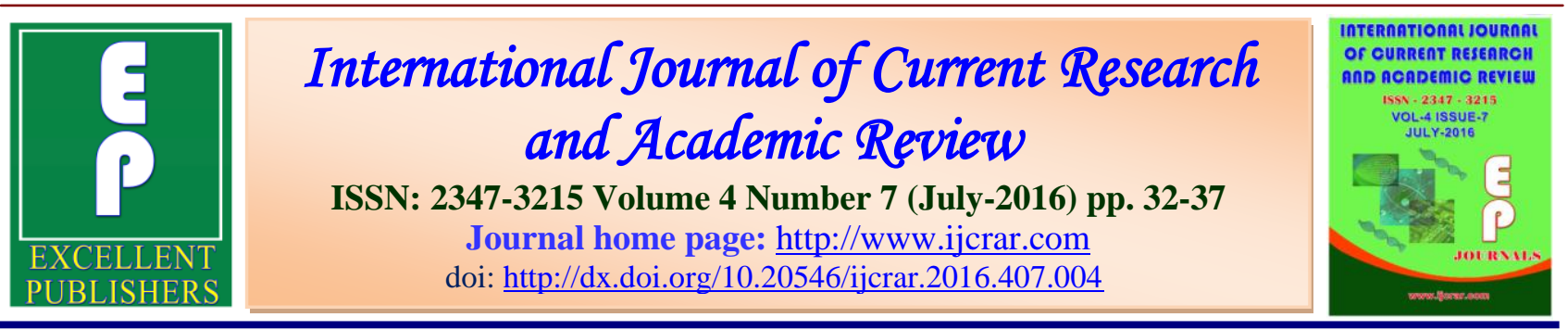

\title{
Evaluation of Clinical Outcome, Drug Utilization and Effect on Liver Enzymes of Anti-epileptics in Epileptic Patients
}

\author{
Mathew George ${ }^{1}$, Lincy Joseph ${ }^{2}$, Robert Mathew ${ }^{3}$ and Preethi Christina Jose ${ }^{4}$. \\ ${ }^{1}$ Department of Pharmacology, Pushpagiri College of Pharmacy, Thiruvalla, Kerala, India \\ ${ }^{2}$ Department of Pharmaceutical Chemistry, Pushpagiri College of Pharmacy, Thiruvalla, \\ Kerala, India \\ ${ }^{3}$ Department of Neurology, Pushpagiri College of pharmacy, Thiruvalla, Kerala, India \\ ${ }^{4}$ Department of Pharmacy Practice, Pushpagiri College of Pharmacy, Thiruvalla, Kerala, India \\ *Corresponding author
}

\begin{tabular}{|l|l|}
\hline \multirow{2}{*}{$\begin{array}{l}\text { KEYW } \\
\text { AST }\end{array}$ ALP, } \\
$\begin{array}{l}\text { Seizure } \\
\text { Semi-auto analyzer, } \\
\text { Colorimetry }\end{array}$ & $\begin{array}{l}\text { Anti-epileptic drugs of various classifications are used to treat different types } \\
\text { of seizures. A prospective experimental study is done in } 50 \text { epilepsy patients } \\
\text { taking atleast } 1 \text { anti-epileptic drug in a tertiary care hospital, Kerala, India. } \\
\text { The objective of the study is to determine the clinical outcome and drug } \\
\text { utilization of anti-epileptics along with the effect of anti-epileptics on serum } \\
\text { liver enzymes such as ALP, AST and ALP. Clinical outcome of the patients } \\
\text { were obtained using determining the seizure control obtained by the patients. } \\
\text { Drug utilization is determined using prescription analysis. Effect in liver } \\
\text { enzymes is evaluated using collecting residual blood sample of the patients } \\
\text { and determined using semi-auto analyzer and colorimetry. }\end{array}$ \\
\hline
\end{tabular}

\section{Introduction}

Epilepsy is one of the common neurological disorder. It is defined as a recurrent, usually unprovoked epileptic seizure which results from excessive synchronous and abnormal firing patterns of the cerebral cortical neuron. Mortality rate among epilepsy patients has been shown to be as high as 90.9 per 1000 person per year. The most common cause of mortality in epilepsy patients has been febrile convulsions (44.9\%). Other causes of death associated with seizures include chest complications (6.8\%), drowning (3.2\%), asphyxia (2.3\%), status epilepticus (2.2\%) and burns (0.8\%). However, management of epilepsy has been shown to be successful with antiepileptic agents.

Most interesting is that $60 \%$ of treated adults stop taking medication without relapse within 2-5 years of treatment. Therefore, with appropriate drug management, improved quality of life in epileptic patients can be achieved (Nurulumi Ahmad et al., 2013). 
Standard treatment provides control of seizures in more than $80 \%$ of patients (Kashinath Gumma et al., 2014). Effective prolonged and specific treatment of anti epileptic drug becomes absolutely essential for successful management. Control of seizures can be achieved through medication adherence. More than half of epilepsy patients have poor seizure control due to non adherence to medication. Effects of non adherence have also been linked to other problems (Nurulumi Ahmad et al., 2013). Drug-induced liver injury associated with antiepileptic drugs (AED) is well recognized. Monitoring or liver enzymes has to be done by liver function tests including tests for ALT, AST, alkaline phosphatase.

Medication adherence is important, especially in chronic disease patients. Adherence to medication is affected by various patient factors such as age, duration of disease and co-morbidities. The direct effect of non adherence with reducing the quality of life in epilepsy patients however is a concern. Therefore, this work aims to identify the level of adherence to medication and perceived quality of life in epilepsy patients in the local setting (Nurulumi Ahmad et al., 2013). This study aims to investigate the extent to which medication adherence, measured using a validated selfreport questionnaire is associated with good seizure control in limited dose.

Different studies on the effect of anti epileptics on liver profile have reported contradictory results. The present study was designed to evaluate significance of liver enzymes in patients of epilepsy when treated with long term anti-epileptic therapy.

\section{Materials and Methods}

The present study was conducted at the Department of Neurology, Pushpagiri
Medical College Hospital, Thiruvalla, Kerala, in which 50 epileptic patients were included. Patients with acute abdominal or hepatic disease (confirmed by USG Abdomen), renal disease, alcohol abuse, organophosphate poisoning and those receiving medications which could alter liver function tests were excluded from the study. All the Patients were subjected initial physical examination and various biochemical investigations initially and after 6 months of continuous treatment with antiepileptics. The study was approved by the Institutional Ethics committee, and informed consent was obtained from all subjects.

Based on customized questionnaires, complex demographic data and clinical parameters were collected:

Age, weight, disease duration, gender, education, type of epilepsy, etiological factors, type of seizures, number of seizures, antiepileptic drugs (AED) administered, its dose and dosage form. For control of seizures, "good control" is defined by an absence of seizure activity since prior visit; "fair control" is defined by one seizure since last visit; and "poor control" is defined by more than one seizure since last visit.

Morisky's medication adherence scale (MMAS) was administered on patients to assess the medication adherence behaviour. The MMAS have four questions of yes/ no type. One point will be given to each 'Yes' answer. Higher score indicates low adherence.

The serum liver enzymes are checked by collecting the residual blood of the patients taking anti-epileptics and using semi autoanalyser alkaline phosphatise is determined and by colorimetry method AST and ALT values are detected. This is done to check the effect of anti-epileptics in liver of 
patients taking anti-epileptic drug for longterm use.

In semi-automatic analyzer using the alkaline phosphates kit, reagent $1,500 \mu l$ and reagent $2,120 \mu \mathrm{l}$ is mixed in a test tube and then the serum sample $10 \mu \mathrm{l}$ is added and incubated for 50 seconds. Then detected in semi-auto analyzer.

In colorimetry, SGOT and SGPT are detected using their respective kits. Absorbance of test, test blank, standard and standard blank are obtained. AST and ALT are calculated using equation:

$$
\begin{aligned}
& \text { AST }=\frac{\text { Abs Test- Abs of test blank }}{\text { Abs Std- Abs of std blank }} \times 200 \\
& \text { ALT }=\frac{\text { Abs Test- Abs of test blank }}{\text { Abs Std- Abs of std blank }}
\end{aligned}
$$

The data on demographics like were analysed by using Microsoft excel of Microsoft operating system windows 2007. Standard deviation, mean and ' $p$ ' value are determined by Chi square test using software.

\section{Results and Discussion}

\section{Socio-demographic characteristics}

A total of 50 patients from in-patient and out-patient department were included in the prospective observational study. The demographic data revealed that the number of male and female patients were $30(60 \%)$ and $20(40 \%)$ respectively.

Maximum number of patients enrolled was in the age group of 39-59 years that is about $38 \%$ and of age group 18-38 and above 60 years was about $36 \%$ and $26 \%$ patients respectively.

Type of epilepsy diagnosed in patients include Generalised tonic-clonic seizure,
Complex partial, Atonic seizure, Simple partial seizure, symptomatic seizure.

\section{AED Utilization pattern}

Monotherapy was commonly used in the management of seizure which accounted $58 \%(29)$, followed by dual therapy, $32 \%(16)$. The least commonly used was treatment that included 3 drugs which accounted about $10 \%$ (5) from the total patients. The most common drug that is used in monotherapy was phenytoin that is about 28 patients use phenytoin, followed by levetiracetam by 18 , sodium valproate by 11 , clobazam by 11 , oxcarbazepine by 3 , phenobarbitone by 1 and carbamazepine by 3.The dose range in $\mathrm{mg} /$ day followed in the patients are:

Phenytoin 100-300mg

Levetiracetam 500-1000mg

Sodium valproate- $200-500 \mathrm{mg}$

Clobazam- 10-20 mg

Oxcarbazepine- 600mg

Carbamazepine- 400mg

\section{Definition of control}

a.Good control is characterized by an absence of seizure activity since prior visit.

b. Fair control is characterized by one seizure since last visit.

c. Poor control is characterized by more than one seizure since last visit.

From the data, it was found that 32 patients were in good control, 11 patients were in fair control and 7 patients were in poor control.

\section{Medication Adherence}

Based on MMAS-4, 36 patients were highly adherent, 10 patients were medium adherent and 4 patients were low adherent. 
Medication adherence of patients has increased significantly with drug use with $\mathrm{p}$ value 0.002 .

\section{Effect on serum liver enzymes}

On use of anti-epileptics some patients developed serum liver enzyme inducing effect from the base-line level. Liver enzymes like ALP, AST and ALT are evaluated. The mean value of AST after drug use is $28.280 \pm 10.0733$ and the $p$ value is 0.008 which is significant. Mean value of ALT after drug use is 32.200 \pm 12.1151 and the $\mathrm{p}$ value is 0.008 which is significant. $\mathrm{P}$ value of ALP was 0.001 with a mean value of $121.360 \pm 18.2519$.

Side effects

Certain side- effects like dizziness, rashes, mouth sore, dyspepsia, vomiting developed in 14 patients using AED.
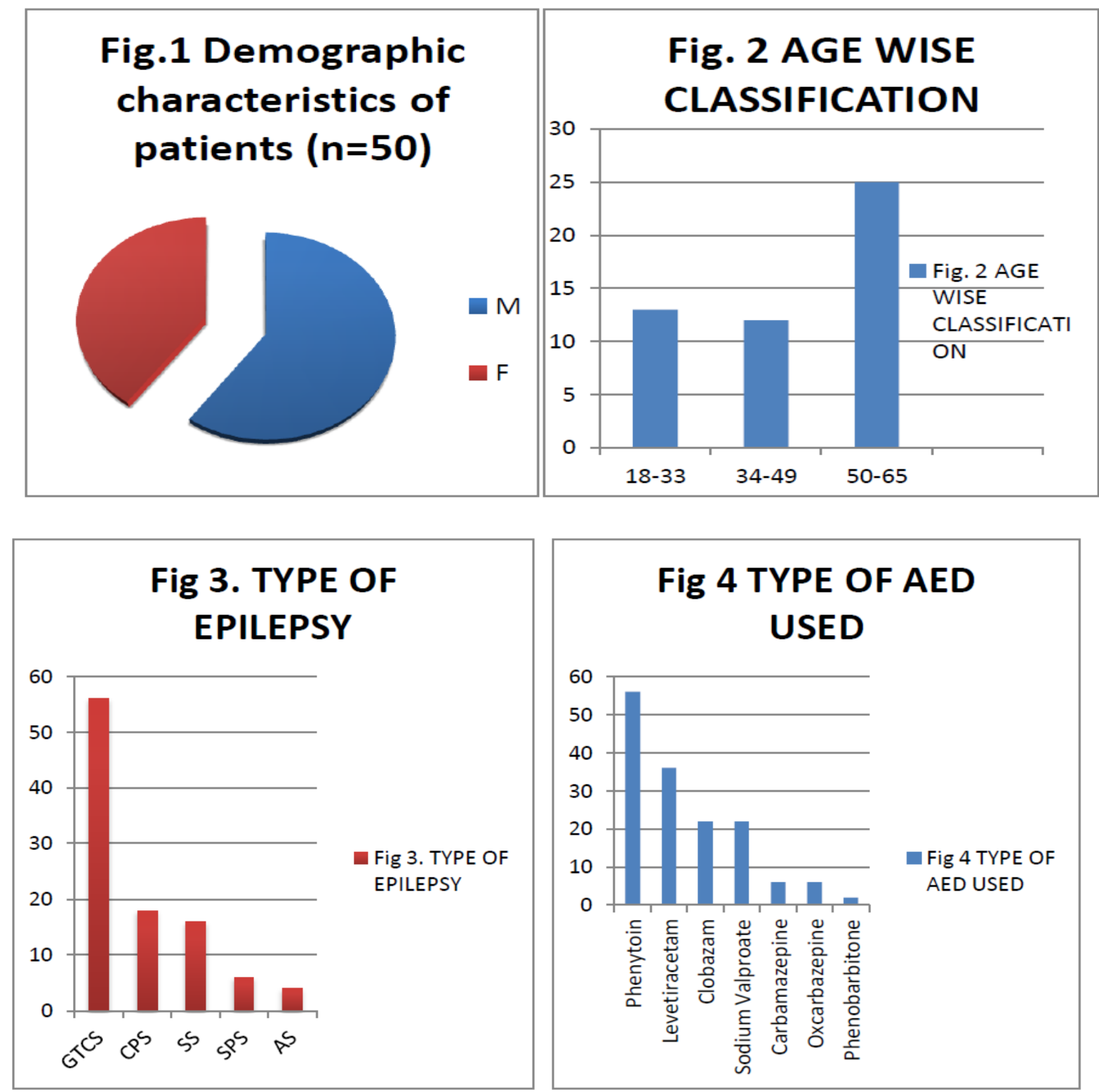

Fig 3. TYPE OF EPILEPSY

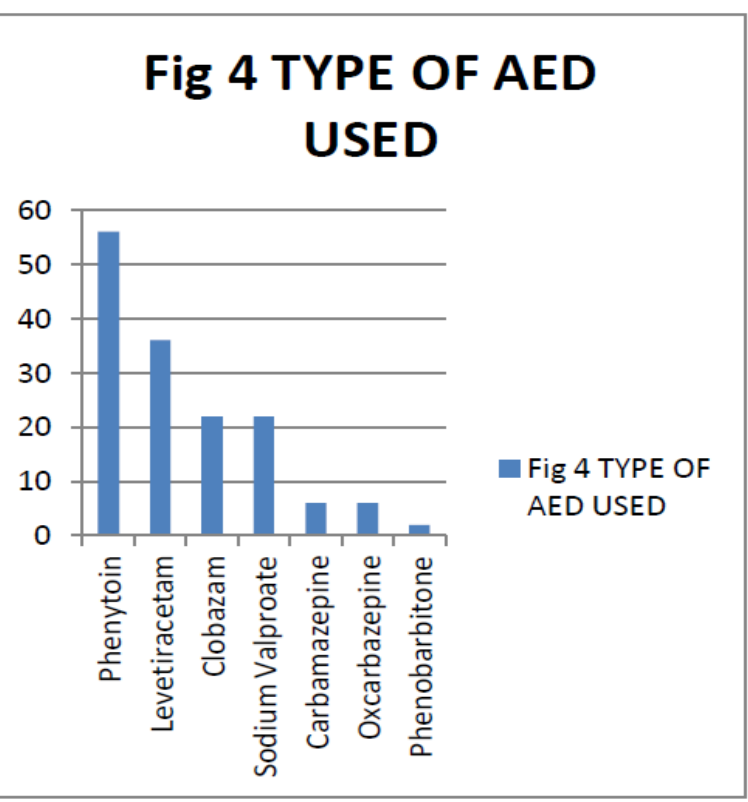




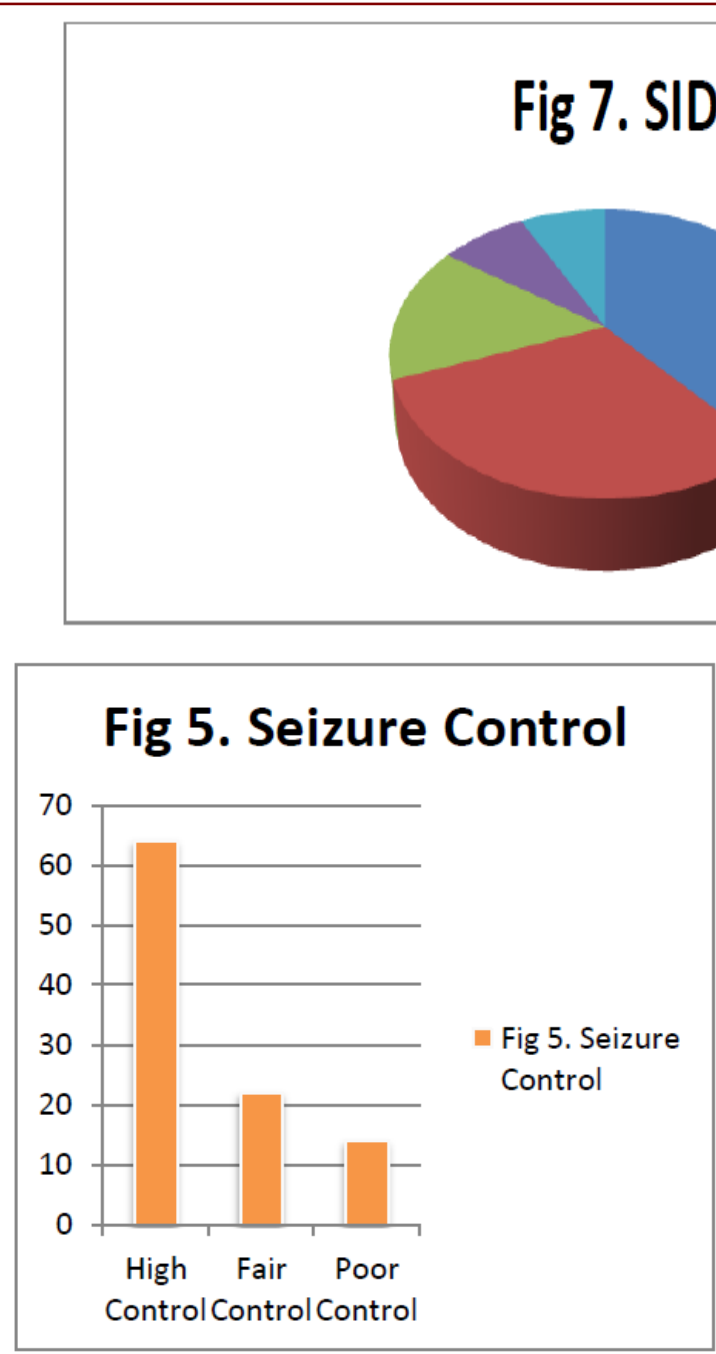

\section{Fig 7. SIDE EFFECTS}

DIZZINESS

RASH

MOUTH SORE

DYSPEPSIA

VOMITING

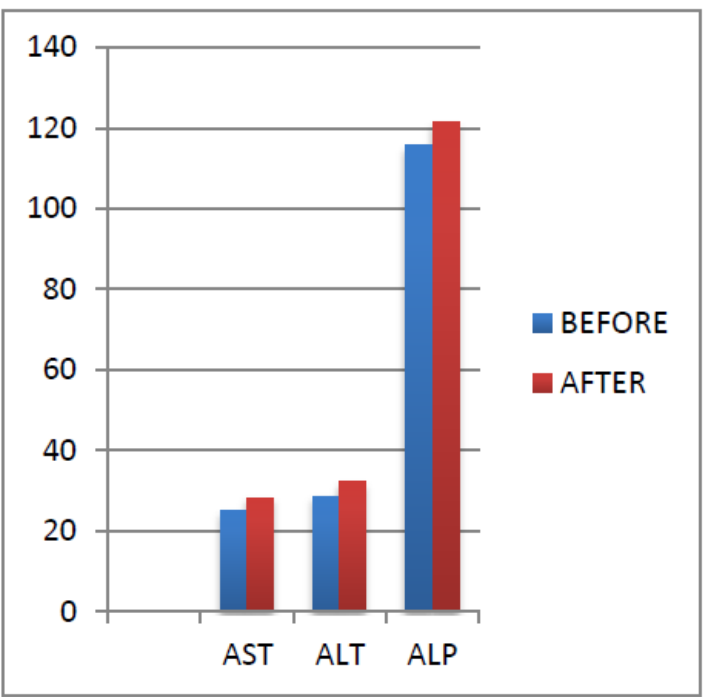

Fig 6. Effect of AED on liver enzyme

According to this study result, GTCS was the most common type of epileptic seizure encountered which accounted about $56 \%$. This result is different from a study done by Mesfin et al(3), on drug use evaluation (DUE) of AEDs at a multispecialty tertiary care teaching hospital in India in which GTCS is about $80 \%$.

Secondly, in this study good control is seen for $64 \%$ patients followed by fair control for $22 \%$ patients and $14 \%$ patients have poor control whereas in a study conducted by Arulkumaran et al., (2009), on drug use evaluation of AEDs, good control was seen in $67 \%$ patients, fair control in $14 \%$ patients and poor control in $17 \%$ patients. In a study conducted by Darmesh et al., on liver enzyme activity during sodium valproate therapy in epilepsy patients showed significant increase in level of aminotransferases whereas in a study conducted by Kashinath et al (2014), on effect of phenytoin sodium on liver enzymes, level of alkaline phosphatise increased significantly. In this study, level of Alkaline phosphatase and aminotransferases increased significantly. Medication adherence of patients after patient counselling is found to be increased significantly in this study due to which high control in seizure is achieved. 


\section{Conclusion}

In this study GTCS was the most prominent seizure encountered. The most common side effect patients faced while the follow-up period was dizziness. The most commonly prescribed AED was phenytoin, followed by levetiracetam. High control in seizure was attained by $64 \%$. Medication adherence in patients increased significantly after patient counselling. The effect of AEDs on liver enzymes such as ALT, AST, ALP is that AEDs have a significant effect on serum liver enzyme induction. Therefore even though there is high seizure control in epilepsy patients after AED use, the patients should be considered for liver function tests.

\section{References}

Darmesh Gamit, Hariom Sharma, Nitinkumar Chaudhary, Nikunj Modi, Kalpana Gamit. Liver enzymes activity during sodium valproate therapy in patients of epilepsy; Int. J. Res. Med. Sci.

Arulkumaran, K.S.G., S. Palanisamy, A. Rajasekaran. 2009. A study on drug use evaluation of anti-epileptics at a multispeciality tertiary care teaching hospital; Int. J. Pharm. Tech. Res., Vol1(4): 1541-1547.

Kashinath Gumma, Gajnan, P., Kulkarni, Padmanabha, T.S. 2014. Effect of Phenytoin Sodium on Liver Function Tests; Int. J. Pharma and Biosci., Vol.5(1): 249-252.

Mesfin Gurshaw, Asrat Agalu, Tesfahun Chanie. 2014. Anti-epileptic drug utilization and treatment outcome among epileptic patients on follow-up in a resource poor setting; J. Young Pharmacists, Vol 6(3): 47-52.

Nurulumi Ahmad, Nurul Izatyothaman, Farida Islahudin. 2013. Medication adherence and quality of life in epilepsy patients; Int. J. Pharm. Pharmaceutical Res., Vol.5(2): 401404.

\section{How to cite this article:}

Mathew George, Lincy Joseph, Robert Mathew and Preethi Christina Jose. 2016. Evaluation of Clinical Outcome, Drug Utilization and Effect on Liver Enzymes of Anti-epileptics in Epileptic Patients. Int.J.Curr.Res.Aca.Rev.4(7): 32-37. doi: http://dx.doi.org/10.20546/ijcrar.2016.407.004 